alkaloids, and the efforts of the American investigators have been concentrated on a study of the pharmacology of a wide range of morphine derivatives and of numerous classes of synthetic compounds having structural features in common with some of those of the morphine molecule.

The chemotherapy of tubercular infections, Prof. Cook said, is now receiving increasing attention. Sir Robert Robinson has recently summarized progress made in this field, pointing out that in tuberculosis, as in leprosy, a suitable therapeutic agent should provide a means of penetrating or breaking down the waxy envelope which surrounds the bacilli of these infections. The active component of chaulmoogra oil, long used in the treatment of leprosy, is known to be a cyclopentenyl fatty acid. Many synthetic analogues of chaulmoogric acid also possess leprocoidal activity, and the nature of these substances gives support to the view that they owe their activity to a capacity to effect impairment of function of the fatty envelope of the organisms.

Prof. Cook also reviewed in the course of his lecture the progress made in the past ten years in the chemistry of the vitamins and the members of the sterol class, including several groups of hormones. Most of the known vitamins, he said, have been isolated in a state of chemical purity, and the principal ones have been prepared synthetically; in addition, the biological role of several of them has been partially elucidated. The pure crystalline vitamin $\mathrm{A}$ was isolated for the first time only a few months ago. The other principal fat-soluble vitamin, known as vitamin $\mathrm{D}$, is now available commercially in chemically pure form. Ascorbic acid, or vitamin
$\mathrm{C}$, is now available as a synthetic product. Incidentally, it has been claimed that vitamins $\mathrm{A}$ and $\mathrm{C}$ increase the body's resistance to infection ; this, said Prof. Cook, is now disputed, although it seems likely that vitamin $A$, by maintaining the tone of the mucosa, renders these less liable to attack by pathogenic organisms. The principal members of the vitamin $B$ complex have been synthesized and considerable insight gained into their biological function. Two other vitamins which have yielded their secrets to chemical investigation in recent years are vitamins $\mathrm{E}$ and $\mathrm{K}$. The availability of the pure synthetic vitamins will hasten the solution of problems concerned with their biochemistry and mode of physiological action.

Remarkable progress has also been witnessed in the chemistry of a group of hormones which are related in molecular structure to each other and to cholesterol, which, Prof. Cook said, may be regarded as their biochemical progenitor. Except in the case of the ostrus-producing hormone, total synthesis has nat yet been achieved, but member's of these groups have been converted into common degradation products, and the steroid hormones have all been prepared artificially from cholesterol or other sterols. Deoxycorticosterone, the most active of the lifemaintenance hormones of the adrenal cortex, has been isolated from ox adrenals and is now prepared artificially in considerable amount. It is concerned in the maintenance of the sodium chloride balance in the blood and has been found effective in the treatment of wound shock; for this reason its availability at the present time is of particular importance.

\title{
CONTROL OF INFECTION IN WAR WOUNDS
}

$\mathrm{T}$ $\mathrm{HE}$ treatment and care of war wounds is essentially a bacteriological problem, as, indeed, experience in the War of 1914-18 made evident. The chief lethal micro-organisms that are found in infected wounds are Streptococcus pyogenes and the group of anærobic bacilli which cause gas gangrene. Researches within recent years into the causation of puerperal fever have shed light upon the origin of pathogenic streptococci in war wounds; in both cases the chief source of infection is regarded as being droplets of secretion that contain these microbes and that are expelled from the throat of those who nurse and treat the patients; the measure of the risk is given in the statement that $2-5$ per cent of adults harbour Streptococcus pyogenes in the throat. Suitable masks should, therefore, be worn by all those who dress war wounds.

The danger from the intrusion of streptococei into wounds is likely to be much lessened by the administration of drugs of the sulphonamide group, although further experience is needed to define clearly the limits of their usefulness. The prophylaxis of gas gangrene is best attained by the early and adequate surgical cleansing of the wound and by the use of specific antitoxins corresponding to the chief types of pathogenic anærobe. The precise role of chemotherapy in combating infections caused by the gasgangrene group of bacteria is still undetermined; recent experiments with laboratory animals indicate that the most favourable results will be obtained from combined sulphapyridine and antitoxin treatment. Tetanus antitoxin, which confers a passive immunity, still retains its place as an invaluable prophylactic agent ; active immunization with tetanus toxoid-a non-toxic modification of tetanus toxinwas adopted last year as a voluntary method of protection for men in the Army and the Royal Air Force.

A small book on the subject, edited by W. H. Ogilvie and modestly styled a war primer ("War Primer on Wound Infection" : its Causes, Prevention and Treatment. By W. H. Ogilvie, Robert Cruickshank, Lawrence P. Garrod, L. E. H. Whitby and G. A. H. Buttle. Pp. 96. (London: The Lancet, 1940.) 2s. $6 d$. net.) is the work of five recognized authorities, each of whom deals with a particular aspect of the treatment of civil and military wounds. The discussion is arranged under the chapter-headings : the problems to be solved; biological aspect ; bacteriology ; antiseptics ; chemotherapy; surgical principles; and surgical procedures. The present state of knowledge has been set forth by the authors in a succinct and wellbalanced manner. The facts they state and the conclusions they have reached deserve to be studied with the greatest care by everyone whose duty it is to assist in treating war wounds. 